\title{
Single lens system for forward-viewing navigation and scanning side- viewing optical coherence tomography
}

\author{
Tyler H. Tate ${ }^{\mathrm{a}}$, Davis McGregor ${ }^{\mathrm{b}}$, Jennifer Barton ${ }^{\mathrm{a}, \mathrm{c}}$ \\ ${ }^{\mathrm{a} C}$ College of Optical Sciences, University of Arizona, 1630 E University Blvd, Tucson, AZ 85721 \\ ${ }^{\mathrm{b}}$ Aerospace and Mechanical Engineering, 1130 N. Mountain Ave, Tucson, AZ 85721 \\ ${ }^{\mathrm{c}}$ Biomedical Engineering, University of Arizona 1657 E Helen Street, Tucson, AZ 85721
}

\begin{abstract}
The optical design for a dual modality endoscope based on piezo scanning fiber technology is presented including a novel technique to combine forward-viewing navigation and side viewing OCT. Potential applications include navigating body lumens such as the fallopian tube, biliary ducts and cardiovascular system. A custom cover plate provides a rotationally symmetric double reflection of the OCT beam to deviate and focus the OCT beam out the side of the endoscope for cross-sectional imaging of the tubal lumen. Considerations in the choice of the scanning fiber are explored and a new technique to increase the divergence angle of the scanning fiber to improve system performance is presented. Resolution and the necessary scanning density requirements to achieve Nyquist sampling of the full image are considered. The novel optical design lays the groundwork for a new approach integrating side-viewing OCT into multimodality endoscopes for small lumen imaging.
\end{abstract}

KEYWORDS: Endoscope, Optical Coherence Tomography, Fluorescence, Cancer, Ovary, Bile Duct, Piezo Scanning, Rotationally Symmetric Reflection

\section{INTRODUCTION}

Forward-viewing endoscopes utilizing piezo scanning fiber (SF) endoscope technology have recently been demonstrated ${ }^{1-3}$. SF is an alternate method of image formation compared to the use of an imaging coherent fiber bundle (CFB) or a miniature detector in the endoscope tip. In one implementation, a single mode fiber is coaxially centered inside a quartered piezo tube. The fiber is fixed to one end of the tube with a short, cantilevered length of fiber protruding (Figure 1). Electrically driving the piezo tube at the mechanical resonance frequency of the fiber causes the cantilevered fiber tip to oscillate. By driving the piezo tube in two orthogonal axes at resonance while controlling the phase difference and amplitude it is possible to scan the fiber tip in a predetermined pattern. A spiral scan is typical, but Lissajous patterns have also been used ${ }^{3}$. The tip of the fiber approximates a point source with Gaussian beam output. Rapidly scanning the fiber in a spiral pattern effectively creates a disc of point sources. An optical illumination system can be designed to image the scanning fiber onto the sample. Calibration allows a priori knowledge of the illumination scanning location as a function of time. High temporal recording of the reflectance or fluorescence light returning from the sample allows the intensity of the image to be known as a function of time. Image reconstruction is performed by correlating the known scan locations and detection intensities as a function of time during each scan.

For endoscopes with diameter smaller than a few millimeters, a miniature detector is too large. SF or CFB are the two primary image formation technologies with advantages and disadvantages compared in Table 1. CFB endoscopes can be built smaller than SF endoscopes with CFB options available as small as 1600 elements (pixels) and just 200-micron outer diameter. Depending on configuration, at least one illumination fiber with diameter of 50-125 microns may also be

Endoscopic Microscopy XII, edited by Guillermo J. Tearney, Thomas D. Wang, Proc. of SPIE Vol. $10040,100400 \mathrm{H} \cdot \odot 2017$ SPIE · CCC code: $1605-7422 / 17 / \$ 18 \cdot$ doi: $10.1117 / 12.2271555$ 
necessary. In comparison the smallest demonstrated SF endoscope uses a piezo tube of 450 -micron outer diameter. ${ }^{1}$ Mounting the tube requires some additional space and reflectance or fluorescence configurations typically use a ring of collection fibers around the outside of the lens system. It may be possible to use a dual clad fiber for both illumination and light collection, but it is unclear if this approach provides sufficient light collection in practice. Thus SF endoscopes smaller than $1 \mathrm{~mm}$ total outer diameter may be possible, but have not yet been demonstrated. A SF endoscope with piezo tube of equivalent diameter to a CFB can have 2-4 times the effective pixel density depending on configuration and scan geometry. The scanning fiber does not suffer from the honeycomb artifact of using a CFB as a relay element. The increased pixel density and lack of honeycomb artifact come at the expense of significantly more complicated image acquisition and reconstruction computations. A major potential advantage of SF technology is the possibility to use the SF for simultaneous dual-modality imaging including scanning OCT that is not practical in a small diameter CFB endoscope. Standard CFBs used for image relay do not preserve phase information necessary for OCT (typically individual cores are multimode and core-to-core coupling occurs).

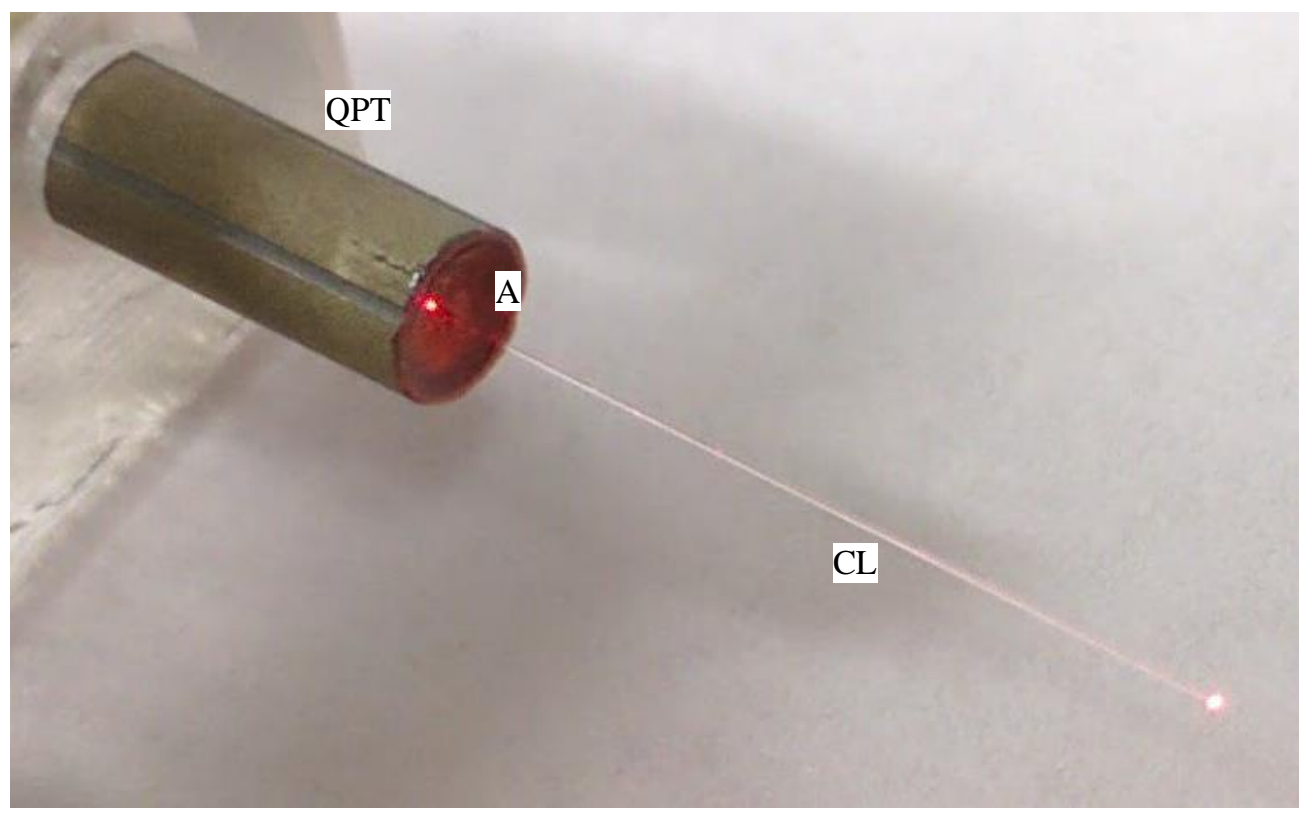

Figure 1: A prototype setup of a quartered piezo tube (QPT) with a cantilevered length of fiber (CL) fixed to one end with adhesive (A) and protruding out. The other end of the QZT is fixed to an acrylic mounting block. A red diode laser is coupled into the fiber.

SF endoscopes have been built for forward viewing using visible light ${ }^{1}$ or $\mathrm{OCT}^{2,4}$. Forward viewing OCT requires a very high speed OCT system in order to create $3 \mathrm{D}$ volume images with good sampling density in real-time. From a practical stand-point, a clinically useful method to display video rate 3D volumes remains a challenge. For certain tubal endoscopic applications, it is more useful to have circumferentially scanning side viewing OCT to create a $2 \mathrm{D}$ crosssectional image of the tubal wall. A forward-viewing reflectance or fluorescence imaging channel is more practical for navigation. Using SF technology, it is possible do design a system that does both side-viewing OCT with scanning and forward viewing navigation.

Most scanning, side-viewing OCT systems rely on a single mode optical fiber with a stationary optical axis and a planar reflecting element. The reflection may be via a mirror, prism or angle polished element. The reflective surface rotates around the optical axis to scan the beam. In an SF design, the output optical axis of the OCT beam can be scanned in a circle around the lens system optical axis. Since the optical axis is no longer coaxial with the lens system, a rotating planar mirror is no longer appropriate for beam turning. In this report, the OCT scan is set to a specific displacement radius of the tip of the cantilevered fiber. The circular scan of the source at the given displacement angle is turned by a rotational symmetric double reflection from a custom cover plate. The result is a circumferential crosssectional scan in the tissue. In this study, the maximal displacement of the fiber is used for the OCT scan. 


\begin{tabular}{|l|l|l|}
\hline Design Feature & CFB & SF \\
\hline Single-channel dual modality imaging & No & Yes \\
\hline OCT scanning & No & Yes \\
\hline UV material transmission challenges & Imaging & Illumination \\
\hline Effective Pixel Density (pixels $\left./ \mathrm{mm}^{\wedge} 2\right)^{1}$ & $\sim 113 \mathrm{k}$ & $\sim 345 \mathrm{k}$ \\
\hline Minimum endoscope diameter & $\sim 500 \mu \mathrm{m}$ & $\sim 1 \mathrm{~mm}$ \\
\hline Imaging honeycomb effect & Yes & No \\
\hline Minimum bend radius of optical fibers & $15-20 \mathrm{~mm}$ & $\sim 2-3 \mathrm{~mm}$ \\
\hline Image processing and reconstruction & Moderate & Complex \\
\hline
\end{tabular}

Table 1: Table comparing design features for dual modality endoscopes based on a CFB and SF design. For UV fluorescence imaging both UV excitation and emission must be transmitted through the system, limiting material choices for the lens system in either configuration. The minimum bend radius of the flexible section of an endoscope depends on the combined characteristics of all materials used in construction. The smaller fibers used in a SF endoscope allow a reduced minimum system bend radius without fiber damage. The rigid tip length in the SF endoscope will typically need to be longer to accommodate the piezo tube and cantilevered fiber.

The forward-viewing channel can be separated from the OCT scan spatially. Since the OCT beam is the maximum displacement, the double reflecting surfaces can be annular such that the forward viewing channel operates at a smaller displacement and transmits through the center of the annuluses. A proof-of-concept optical design for this system is shown in Figure 2.

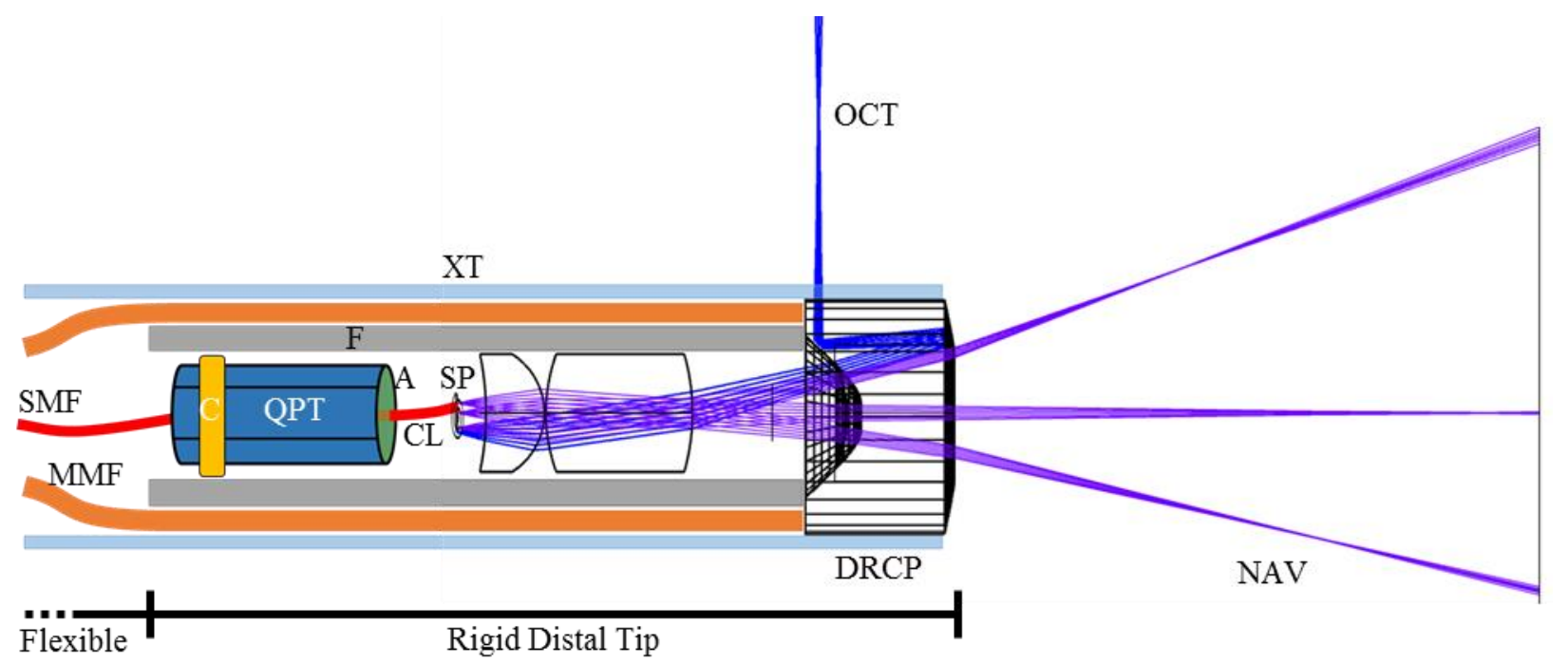

Figure 2: Sample design of a dual modality SF endoscope with side-viewing OCT and forward viewing navigation (NAV). The design shows the single-mode fiber (SMF) passing through the quartered piezo tube (QPT). It is adhered (A) to the tube with cantilevered length (CL) protruding to produce the scan pattern (SP). The QPT is attached to the rigid ferrule $(\mathrm{F})$ with a mounting collar $(\mathrm{C})$. The lenses are also held in by the ferrule. A ring of high NA multimode fibers MMF surround the ferrule. The outer most layer is IR transparent extruded tubing (XT). The double reflection cover plate (DRCP) at the distal end separate the two image modalities spatially. Design is not to scale. 


\section{DESIGN}

A complete conceptual design of a dual modality SF endoscope for side-scanning OCT and forward viewing navigation is presented. This includes a detailed optical design of the endoscope tip and a sample system design. The focus of the report is the choice of scanning fiber and lens design including the novel double reflection cover plate (DRCP) that spatially separates the two channels. A sample of the layout of an endoscope distal tip with a DRCP is shown in Figure 2. For the prototype design, the forward viewing channel is assumed to be monochromatic with $406 \mathrm{~nm}$ illumination suitable for reflectance imaging and excitation of intrinsic fluorophores in tissue. A sample system diagram is shown in Figure 3, assuming a tri-color blue, green, and red illumination system was implemented. The complete system, mechanical and optical design for a dual modality endoscope is multi-faceted and specific design choices depend on the application. This section focuses on the optical design considerations and characteristics including the DRCP.

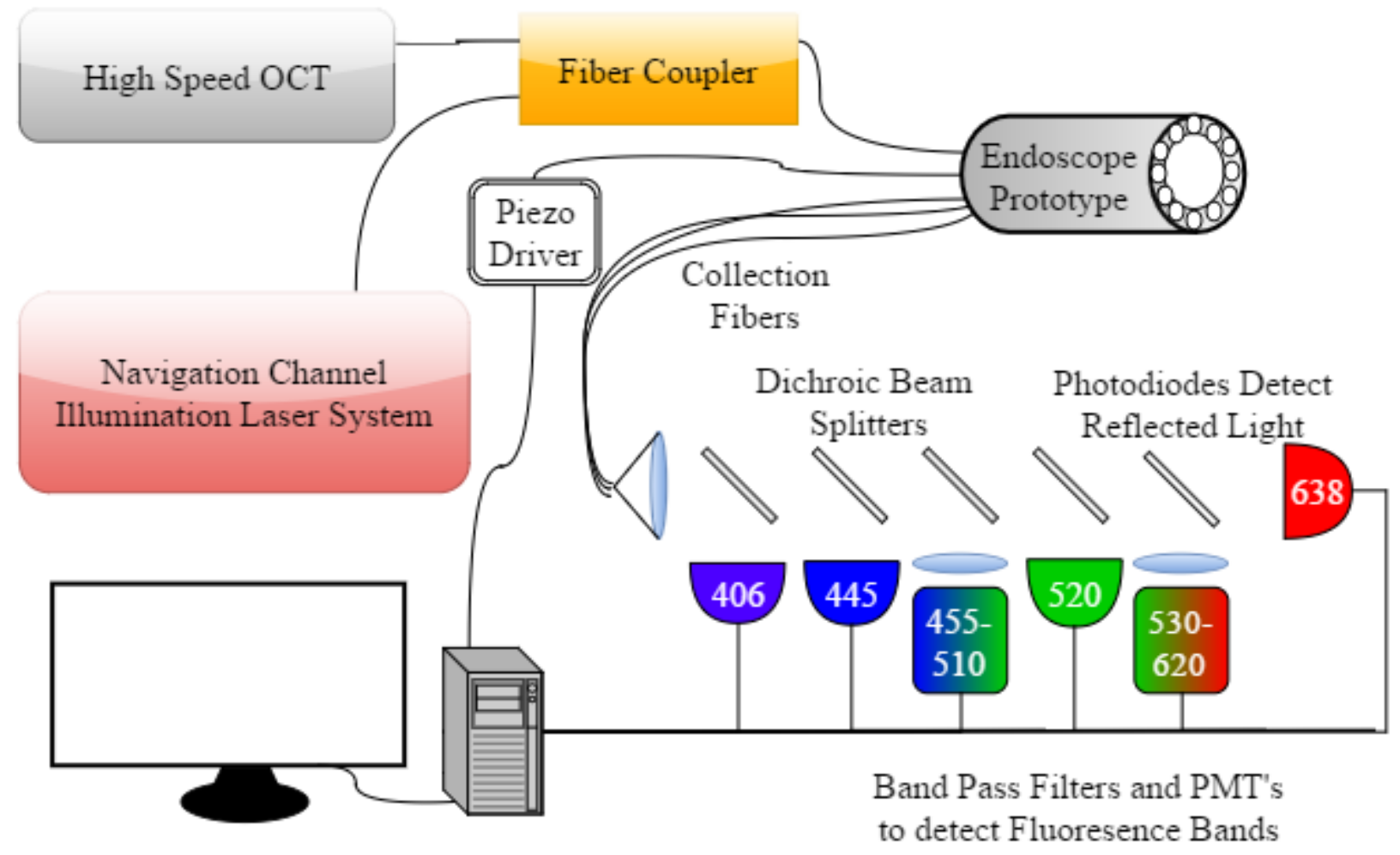

Figure 3: Sample system diagram for a dual modality scanning fiber endoscope. The illumination for the navigational channel and OCT must be combined using a fiber coupler into a single fiber for scanning. In this example, the detection system for the navigation channel includes multiple photodiodes and photomultiplier tubes (PMT) to simultaneously detect reflected light and fluorescence from multiple illumination wavelengths. This can be configured for white-light imaging, fluorescence imaging or both as necessary for the application.

\subsection{Illumination wavelengths}

The choice of illumination wavelengths will impact optical performance, design and material choices. OCT requires a single-mode (SM) fiber operating in the near IR with typical central wavelength around $890 \mathrm{~nm}, 930 \mathrm{~nm}, 1060 \mathrm{~nm}$ or $1300 \mathrm{~nm}$. Longer wavelengths tend to have deeper penetration in tissue, but worse imaging resolution. The DRCP material is an acrylic polymer suitable for diamond turning fabrication. The specific polymer will affect transmission range, but generally transmission efficiency for acrylic has drop outs above $1200 \mathrm{~nm}$. For the proof of concept design a central wavelength of $1000 \mathrm{~nm}$ is used. Slight modifications to the design can be made to accommodate the specific OCT system used. 
The navigation channel will be used for reflectance or fluorescence imaging. For reflectance imaging a single visible laser can be used for monochromatic imaging or a combination of red, green and blue lasers can be used for white-light equivalent imaging. For autofluorescence imaging blue or near UV sources are necessary. The broader the wavelength range the more chromatic considerations are necessary for the optical design and the choice of scanning optical fiber. For this design a single mode fiber coupled $406 \mathrm{~nm}$ fiber diode laser (e.g. Thorlabs LP406-SF20) is assumed to be the source. Reflected light can be used to generate a monochromatic image and the wavelength is short enough to excite many endogenous fluorophores. Simultaneous collection of reflected light and fluorescence emission could be used to create a reflectance image overlaid with fluorescence signals. Additional laser wavelengths could be added in the future for color imaging or multispectral fluorescence imaging (Figure 3).

\subsection{Scanning Fiber}

Fundamentally, the scanning fiber is the light source in an illumination system. Before designing the lens system to image the light source into the sample space it is critical to know the light source characteristics. In particularly the numerical aperture (NA) of the fiber and the scanning geometry will determine the achievable limits to the resolution and FFOV of the illumination system.

OCT is an interferometric technique and requires a SM fiber at the OCT wavelengths to preserver phase information. The navigation channel does not require a SM fiber, but the even illumination of an SM fiber is preferred. The mode field diameter (MDF) of a SM fiber is only SM over a limited wavelength range, typically of a few hundred nanometers in the red and near IR spectrum. The operable SM bandwidth decreases as the central wavelength shortens. The $V$ number is a dimensionless parameter associated with step index fibers defined as:

$$
V=\frac{2 \pi}{\lambda} * a * N A(\lambda)=\frac{2 \pi}{\lambda} * a * \sqrt{n_{\text {core }}^{2}(\lambda)-n_{\text {cladding }}^{2}(\lambda)}
$$

In this equation $a$ is the radius of fiber, NA is the numerical aperture, $n_{\text {core }}$ is the index of refraction of the fiber core and $n_{\text {cladding }}$ is the index of refraction of the fiber cladding. Emphasized here is that the indices of refraction and thus NA are wavelength dependent. Fibers only support a single mode of transmission below $V \approx 2.405$. For larger $V$ numbers the number of supported modes is:

$$
\text { Number of Modes } \approx \frac{V^{2}}{2}
$$

This becomes relevant if a SM fiber designed for 1 micron wavelengths is also used with $406 \mathrm{~nm}$ wavelengths. With the rough assumptions that the $V$ number for a SM fiber is 2 for 1 micron light and the NA is constant than the $V$ number will be around 4.9 for $406 \mathrm{~nm}$ light and thus allow approximately 12 modes of propagation. The output power distribution will be a super position of propagation modes rather than a disc with smooth Gaussian power distribution. When modeling the output of a SM fiber as a Gaussian beam the MDF is twice the beam waist, $\omega_{0}$, and can be approximated from the $V$ number using 5 :

$$
\frac{\omega_{0}}{a} \approx 0.65+\frac{1.691}{V^{3 / 2}}+\frac{2.879}{V^{6}}
$$

All other parameters of the Gaussian beam including the divergence angle which is equivalent to the NA in the farfield can be derived from the beam waist size. When using a SM fiber with wavelengths shorter than the design specification the fiber becomes multimode and has a different mode field diameter (and thus a different NA). For the navigation channel the practical impact of these effects should be examined experimentally to determine if they lead to an unacceptable degradation of illumination beam quality and thus image quality. If the negative effects are minimal for the forward viewing channel then the simplest solution for a dual modality system is to use a SM fiber designed for the wavelength ranges of the OCT system.

Alternative fiber choices include dual-clad fibers or multi-core fibers. Dual clad fibers have a SM core to transmit the OCT. There are then two layers of cladding and the inner cladding may be suitable for the navigation channel 
illumination. It will still be multimode, but may have many modes and when used in combination with a mode scrambler provide a reasonably even illumination pattern. The inner cladding may also be able to be designed with a higher NA than the SM core allowing for improved resolution in tissue space for the forward viewing channel. A multi-core fiber could also be custom designed in which multiple SM cores are placed in the same fiber. Each core would be SM over a limited wavelength range and in combination could cover any desirably range of the near UV, visible and near IR spectrum. The total size of a dual-clad or multi-core fiber may be larger than what is available as a standard SM fiber. The increased mass will reduce the resonant scanning frequency.

The scanning geometry and maximum deflection of the tip of the fiber relative to the optical axis the lens system defines the physical extent of the illumination source. The maximum deflection depends on the material properties and physical dimension of the piezo tube and the fiber as well as the cantilevered length of the fiber. The equations for determining the resonance frequency of the fiber have eigenvalue solutions of increasing frequency. The maximum tip deflection is achieved with the lowest resonance frequency. Experimentally, a $17 \mathrm{~mm}$ length of cantilevered 780HP fiber has been verified to have a maximum tip deflection of $\pm 200 \mu \mathrm{m}$ with a RMS driving voltage of $20 \mathrm{~V}$ at the lowest resonance frequency. Depending on the specific configuration of the piezo tube and fiber the tip deflection will change. This study assumes $\pm 200 \mu \mathrm{m}$ as a reasonable initial design values. Increasing the maximum tip deflection increases the total number of effective pixels and thus can improve the resolution or FFOV.

\subsection{Increasing the Numerical Aperture}

The lens design initially assumes a SM fiber designed for 1-micron light with an NA of 0.127 (MFD=5). SM fibers operating near 1 micron typically have NA values between 0.1 and 0.2 so these are reasonable, but conservative estimates. For this study the same SM fiber when used with $406 \mathrm{~nm}$ light is assumed to have a reduced NA of 0.086 $(M F D=3)$. This number is an approximation based on the above equations and needs experimental verification. The optical design for both the OCT and navigation channel would benefit from increased fiber NA.

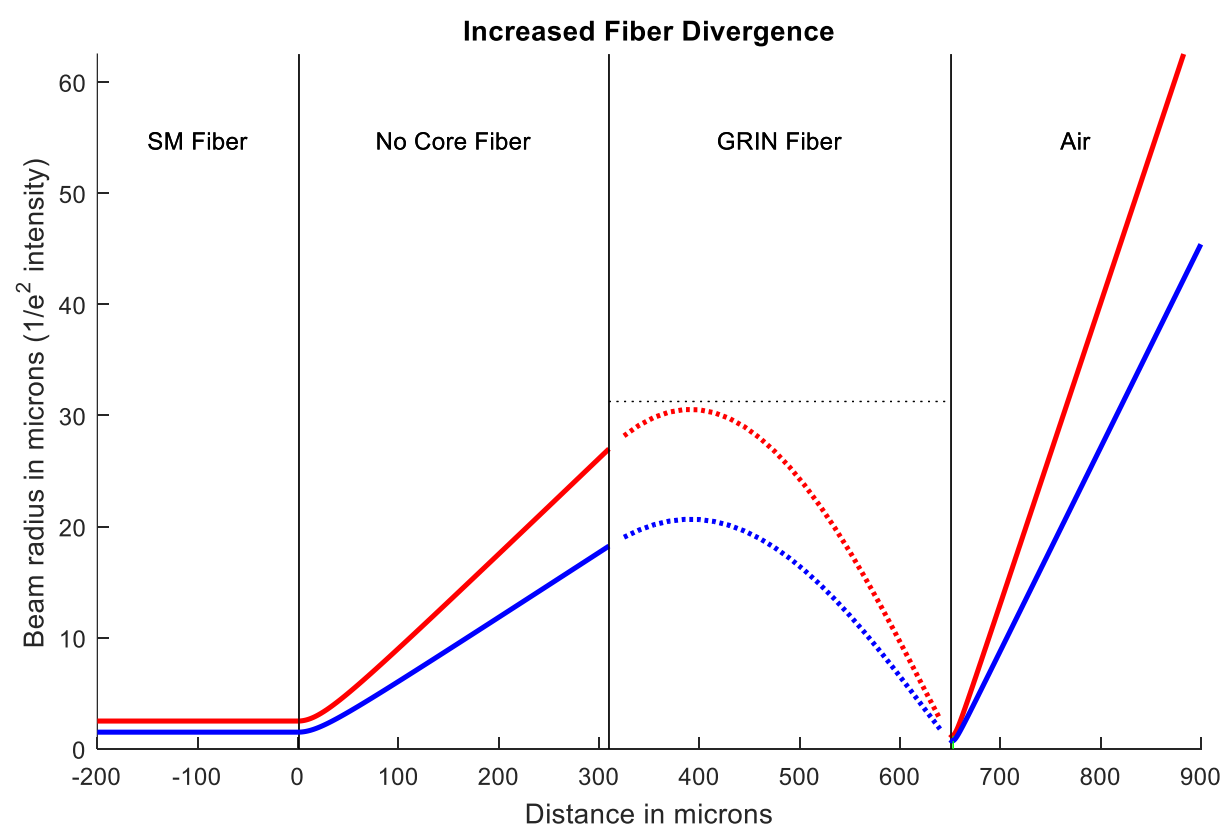

Figure 4: Plot of the beam waist (1/e^2 intensity) vs distance from the output of the SM fiber. A GRIN fiber is used to increase the divergence angle. The red line represents the 1-micron beam with initial MFD of 5 microns and the blue line represents the 406-nm beam with initial MFD of 3 microns. The SM fiber, no core fiber and GRIN fiber are all assumed to have outer diameter of 125 microns and to be fusion spliced together. The horizontal dashed line in the GRIN fiber represents the maximum usable aperture. 
One method of increasing NA coming out of the fiber is to fusion splice a short length of large core multimode fiber followed by a short length of gradient index (GRIN) fiber onto the end of the scanning fiber (Figure 4). The lengths are short enough and the material characteristics and physical dimensions are similar enough to standard SM fibers that the resonant properties of the fiber are not expected to be degraded. The fusion spliced fibers create a very short relay system that reimages the beam waist to a smaller size just outside of the spliced GRIN fiber. The smaller beam waist corresponds to an increased divergence angle after the focal spot. Using this technique, the output NA of the fusion spliced assembly can be increased to 0.27 for the 1-micron light and 0.18 for the $406 \mathrm{~nm}$ light. The technique is ideal for single mode fibers and dual clad fibers as the optical axis and mechanical axis are coaxial. Multicore fibers with each core having an independent optical axis slightly displaced from the optical axis of the GRIN fiber may have minor angular divergence for light from each core at the output.

The majority of the source properties critical to the lens design have now been discussed. One final consideration is that the output optical axis of the oscillating fiber will tilt with respect to the optical axis of the lens system as a function of displacement. For a cantilevered fiber length of $17 \mathrm{~mm}$ a conservative estimate based on early experimental results with the setup shown in Figure 1 suggest that the tangent line from the tip of the fiber would cross the axis $10 \mathrm{~mm}$ behind the fiber tip. Thus the maximally divergent tangent line creates a triangle with a $10 \mathrm{~mm}$ base and 200-micron height. This corresponds to a divergence angle of 1.15 degrees. For the proof of concept endoscope this value is considered negligible. Thus the complete assumptions for the light source are a 200 -micron fiber tip deflection with telecentric output of $0.27 \mathrm{NA}$ at 1 micron and $0.18 \mathrm{NA}$ at $406 \mathrm{~nm}$

\subsection{Lens Design}

The goal of the design is to provide forward viewing navigation with as large of a FFOV as possible with simultaneous side viewing OCT. For applications like transvaginal ovarian cancer screening and biliary duct imaging the endoscope outer diameter is ideally $3.3 \mathrm{~mm}$ or less. Due to the small size and limited alignment compensation of endoscopes, lens designs with few and simple elements are preferred. For this design two glass lenses with spherical surfaces are used in addition to the custom DRCP.

Due to the significant difference in wavelength between the two modalities the lens design functions differently for each wavelength. The first two lenses provide sufficient focal power to focus the $406 \mathrm{~nm}$ light in front of the endoscope. If the cover plate had flat surfaces the $406 \mathrm{~nm}$ light would be focused just outside the cover plate front of the endoscope with nearly 0.4 NA and 17.5 degree FFOV. The first (proximal) surface of the cover plate has negative power to increase the working distance and field of view at the expense of reducing the NA. The rear (distal) surface of the cover plate is flat and transmissive over the region that the forward viewing rays transmit.

The design from the perspective of the forward-viewing channel along with spot diagrams in one millimeter steps for the first 5 millimeters of working distance is shown in Figure 5. The NA is approximately 0.01 corresponding to an airy disk diameter of 44 microns. The FFOV is 30 degrees. The minimal spot size is at progressively shorter working distances as the field angle increases. For illumination inside of a tube this is an advantage. The tubal walls will have higher resolution at shorter working distances while the tubal opening in front of the endoscope will have higher resolution at longer working distances. As an illumination system the working distance can be considered any distance from which sufficient reflected or fluorescence light is collected to generate an image. The illumination beam will have angular resolution with best spatial resolution at the minimum spot diameters. The low NA system will have reasonably consistent performance over a long working distance.

The field of view is limited by the spatial separation of the navigation and OCT beams on the second surface of the cover plate. An annular region is used to reflect the OCT beam limiting the range of deflection usable for navigation to $75 \%$ of maximum. In terms of area, only $56 \%$ of source extent is used leading to significant loss of potential pixels.

For OCT only the largest field angle beam originating from the maximal fiber tip deviation is considered and it takes a different path through the lenses system. The light passes through the first two lenses and the negative power central region of the first surface of the cover plate similar to the navigation channel. The significantly longer wavelength experiences less refractive power at each surface and the beam is approximately collimated after entering the cover plate. 
Rather than pass through the flat transmissive central region on the second surface of the cover plate the OCT beam reflects from an annular region of the cover plate with an aspheric profile. The light makes a second pass through the cover plate and a second reflection from another reflective annular region of the cover plate with an aspheric profile on the first surface (Figure 6). The first reflection primarily adds positive focal power to the beam along with some deviation. The second reflection primarily deviates the beam for side viewing and adds some negative power to extend the working distance and depth of focus.

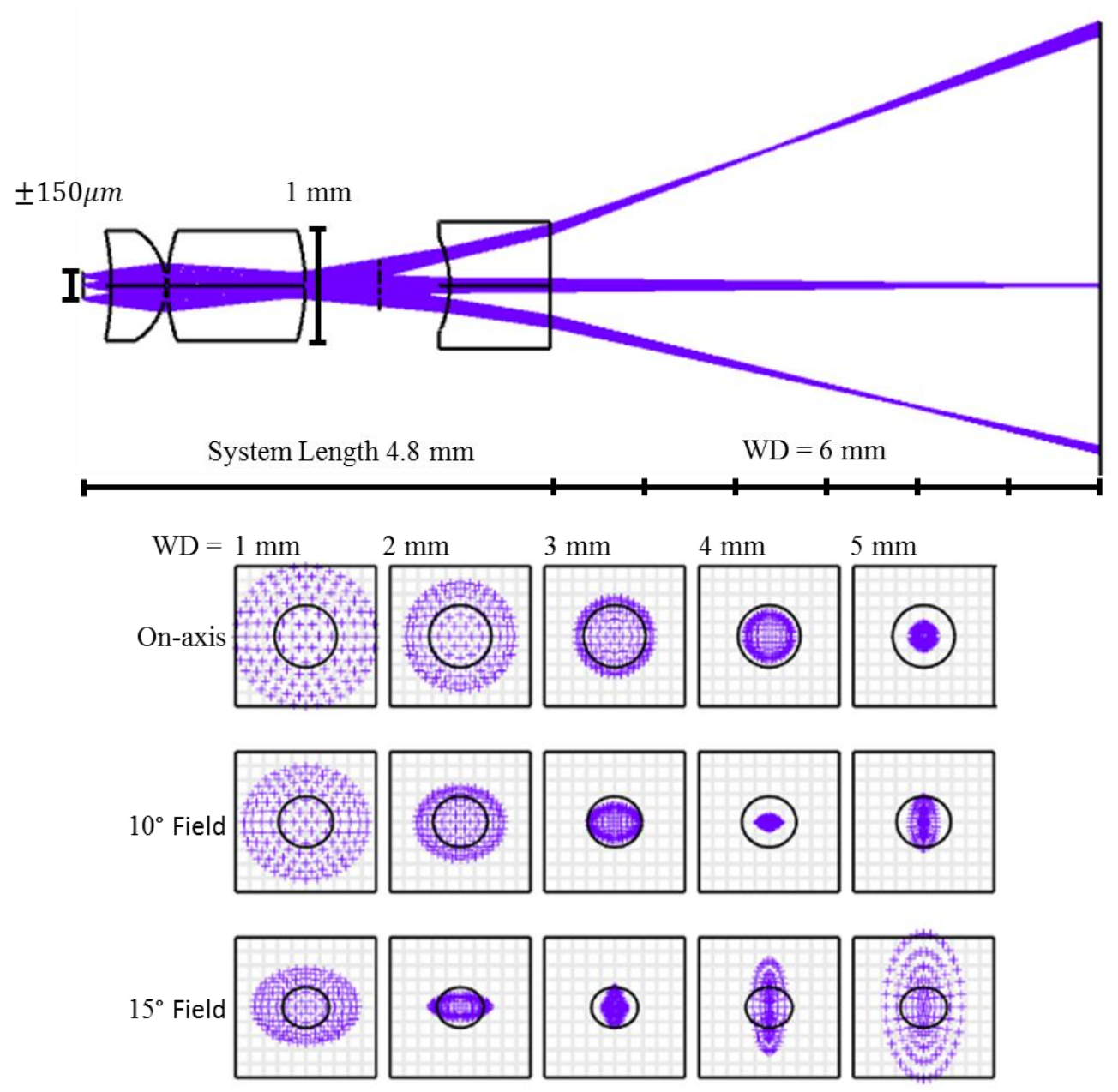

Figure 5: Top - Lens system shown in forward viewing navigation configuration. Only the portion of the cover plate is shown that interacts with the forward viewing rays. Bottom - Spot diagrams for each field in $1 \mathrm{~mm}$ steps from $1 \mathrm{~mm}$ to $5 \mathrm{~mm}$ working distances. Each box has 100-micron side lengths and airy disks are shown in each box with diameter 44.32 microns.

The OCT beam is designed for best focus 1.5-2.5 mm outside the cover plate as shown in Figure 6. A square wave target with $25 \mathrm{LP} / \mathrm{mm}$ has contrast above the Rayleigh limit (9\%) for a range of working distances from $0.5 \mathrm{~mm}$ to 4 $\mathrm{mm}$. In this implementation of the design, the cover plate is $2.4 \mathrm{~mm}$ in diameter leading to best focus in an annular region of 5.4 to $7.4 \mathrm{~mm}$ diameter. For reference, the bile duct diameter is $4.1 \mathrm{~mm}$ in normal patients and can increase to over $11 \mathrm{~mm}$ in abnormal conditions such as gallstones, pancreatitis or tumor development ${ }^{6}$. OCT functions best with the focus inside of the tissue making this design suitable for bile duct imaging. Further increasing the working distance with the same sized DRCP are challenging and result in a loss of resolution. The entire endoscope diameter including DRCP could scale for different applications. Diamond turning can fabricate $1 \mathrm{~mm}$ thick by $1 \mathrm{~mm}$ diameter elements relatively easily. Smaller sizes may pose fabrication and coating challenges. 


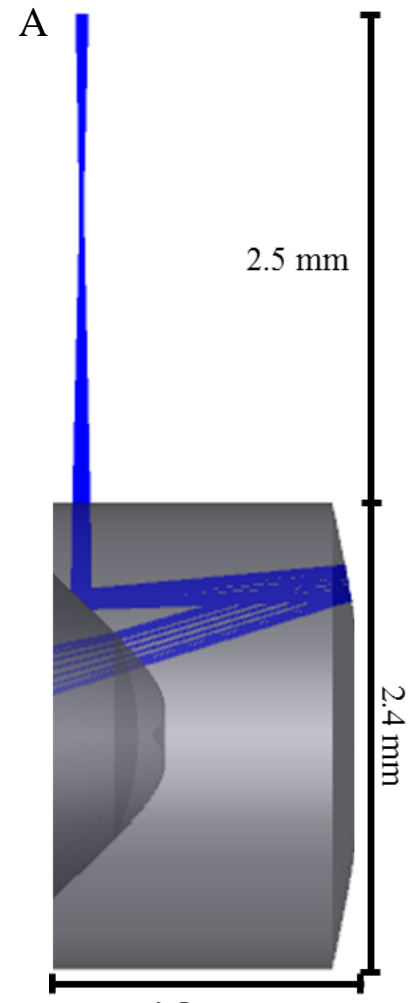

$1.5 \mathrm{~mm}$
$\mathrm{B}$
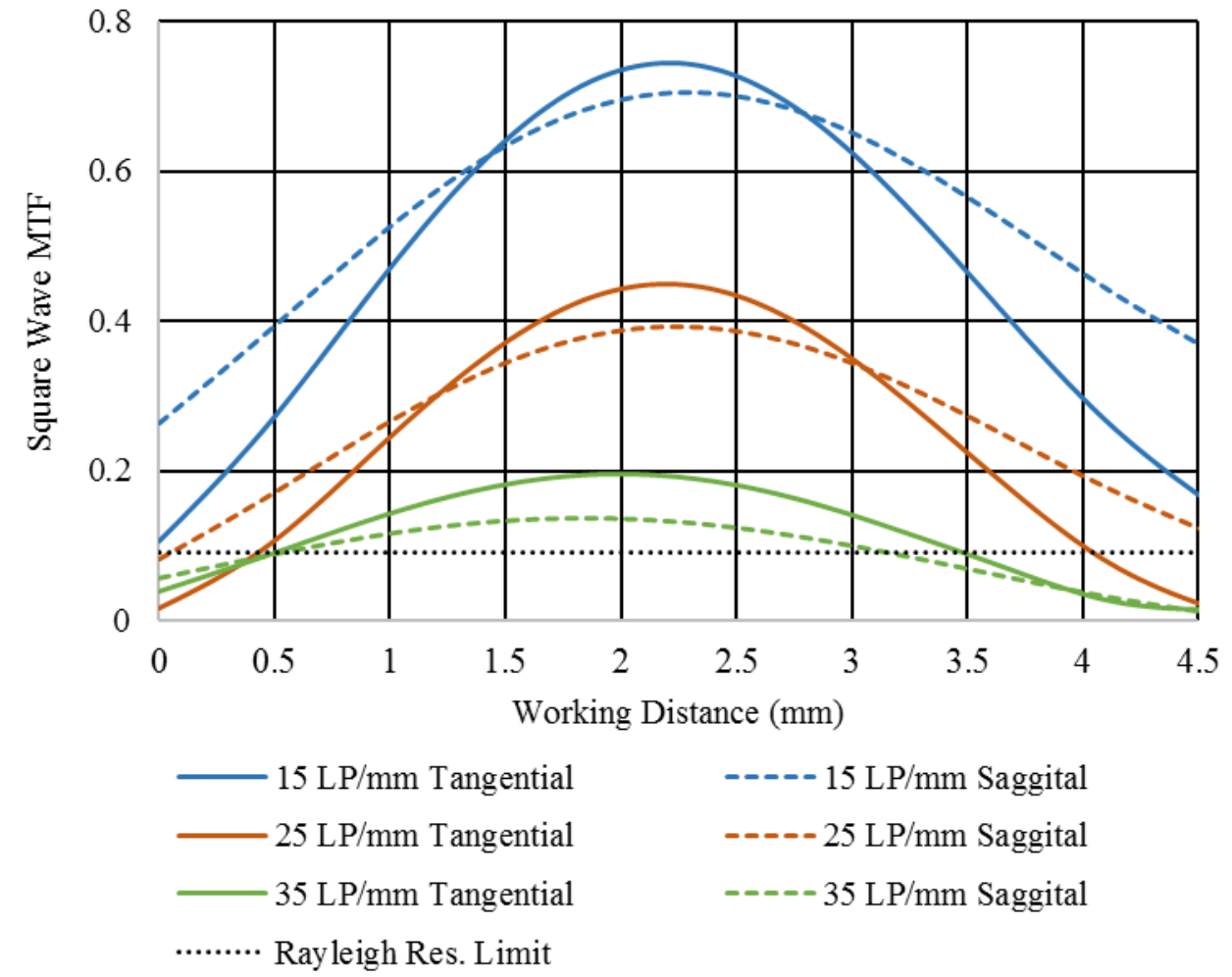

$---15 \mathrm{LP} / \mathrm{mm}$ Saggital

$---25 \mathrm{LP} / \mathrm{mm}$ Saggital

$----35 \mathrm{LP} / \mathrm{mm}$ Saggital

Figure 6: A) OCT light path through double reflection cover plate. B) Graph showing the MTF contrast of a square wave target as a function of working distance for the OCT system. Best focus is generally between 1.5 and $2.5 \mathrm{~mm}$ working distances. Lower frequency square wave targets have higher contrast and larger depth of fields.

\subsection{Resolution Considerations}

Resolution of a scanning fiber endoscope depends on scanning geometry, sample acquisition rate and lens system. The scanning geometry and sample acquisition rate can be adjusted after construction allowing an adjustable trade-off between sampling density and scanning speed. The best achievable resolution is fundamentally limited by the illumination lens system. The lens system is imaging the effective point source of the scanning fiber onto the sample. Assuming a diffraction limited imaging system the Rayleigh resolution, $\Delta x$, is half the airy disk diameter given by:

$$
\Delta x=\frac{D_{\text {airy }}}{2}=\frac{1.22 \lambda}{N A}
$$

The theoretical maximum number of resolvable points is thus equivalent to the number of non-overlapping circles with diameter $\Delta x$ that have centers inside the illumination disc used to create the image with radius $R_{i m g}$. This is a classic circle packing problem and the solution is best approximated with a computational algorithm as there is no explicit formula ${ }^{7}$. Free online computation tools such as WolframAlpha can be used to quickly make the calculation. For the system described the beam is diffraction limited at all field angles at a working distance of $3.8 \mathrm{~mm}$ with $\Delta x=$ $19.05 \mu \mathrm{m}$ and $R_{i m g}=2.032 \mathrm{~mm}$. Given these parameters approximately $41 \mathrm{k}$ resolvable points are possible. The forward viewing channel only uses \pm 150 micron tip deflection of the fiber which corresponds to a scanned area of 0.071 $\mathrm{mm}^{2}$. Thus $41 \mathrm{k}$ resolvable points corresponds to a pixel density of $580 \mathrm{k}$ pixels $/ \mathrm{mm}^{2}$ exceeding the estimated in Table 1 . For comparison, a CFB with 3.1-micron core-to-core separation and a 325-micron diameter image circle (e.g. Fujikura FIGH-10-350S) has 10,000 pixels, which also corresponds to a slightly higher number than estimated in Table 1 with 120,000 pixels $/ \mathrm{mm}^{2}$. An improvement of 3-5 times the pixel density when compared to a CFB is expected. Some degradation will occur in the scanning fiber resolution due to scanning artifacts and digital resampling. However, similar 
image quality degradation is present when using a CFB as the honeycomb structure images onto a digital detector on the proximal side of the endoscope.

For Nyquist sampling, the system must sample with $\Delta x / 2$ spacing. The sampling for a scanning fiber endoscope typically occurs in a spiral pattern and the collected samples must be digitally resampled for display on a Cartesian pixel grid. The final resolution will be determined by the sampling frequency, scan artifacts, optical aberrations and digital resampling efficiency.

The approximate number of sample points per scan for Nyquist sampling can be determined from the scanning geometry. Assuming negligible distortion and other aberrations a spiral scan of the fiber tip will image to a spiral scan in sample space. The parameterized Cartesian coordinates for the sample space spiral scan are given as a function of time by:

$$
\begin{aligned}
& x(\boldsymbol{t})=R(\boldsymbol{t}) * \cos (\boldsymbol{t}) \\
& y(\boldsymbol{t})=R(\boldsymbol{t}) * \sin (\boldsymbol{t})
\end{aligned}
$$

Where $\boldsymbol{t}$ is a time vector for each image acquisition from time zero to total image acquisition time $\mathrm{T}$ and sampling rate $1 / \Delta t$. The radial location of the point is given by $R(t)$ as a function of time with a maximum value equal to $R_{i m g}$. For simplicity this derivation assumes a simple Archimedean spiral in which $R(\boldsymbol{t})$ is a constant function of time (linear). Thus the branches (rings) of the spiral have constant separation distance.

Nyquist sampling requires at least two samples per $\Delta x$. The total number of samples, $N_{\text {Total }}$ can be determined as the product of the necessary number of samples per spiral branch, $N_{\theta}$, and the necessary number of spiral branches, $N_{r}$. With a constant time between samples, $\Delta t$, the number of samples per branch is constant. In other words, the angular separation between each sample point with respect to the origin is constant, but since the radial distance between each sample point is increasing the distance between consecutive sample points increases over time. The sampling density is thus foveated with Nyquist sampling at the edge of the image requiring oversampling the center of the image. Both $N_{r}$ and $N_{\theta}$ can be calculated using $\Delta x$ and $R_{i m g}$ :

$$
\begin{gathered}
N_{\theta}=\frac{\pi}{\sin ^{-1}\left(\frac{\Delta x}{2 R_{\text {img }}}\right)} \\
N_{r}=\frac{2 R_{\text {img }}}{\Delta x} \\
N_{\text {Total }}=N_{r} * N_{\theta}
\end{gathered}
$$

Using the values for the resolution and image size above this system approximately requires $N_{r}=213$ and $N_{\theta}=670$ for Nyquist sampling of the full image. Thus 285,420 samples must be acquired during the full scan. A sample acquisition spiral with $N_{r}=21$ and $N_{\theta}=67$ is shown in Figure 7 .

The time required for each branch of the spiral will be determined by the resonance frequency $\omega_{0}$. The interval between samples and total image acquisition time can be determined from $\omega_{0}$ :

$$
\begin{gathered}
\Delta t=\frac{1}{N_{\theta} \omega_{0}} \\
T=\Delta t * N_{\text {Total }}=\frac{N_{\text {Total }}}{N_{\theta} \omega_{0}}=\frac{N_{r}}{\omega_{0}}
\end{gathered}
$$

For the forward viewing channel to achieve a $24 \mathrm{~Hz}$ video frame rate the resonance frequency must be $5.1 \mathrm{kHz}$ assuming continuous acquisition. In reality, maintaining a highly repeatable scan during continuous scanning may not be possible. Additional time may need to be added after each spiral to reset the fiber velocity to zero using active dampening and passive decay. This can add as much as $40 \%$ additional acquisition time to each image and thus would 
require a resonance frequency of $7.1 \mathrm{KHz}$ in order to maintain Nyquist sampling for $24 \mathrm{~Hz}$ video rate imaging for this design. Depending on the application it may not be necessary to achieve Nyquist sampling of the full image in which case the number of samples may be reduced in exchange for increased frame rate. Ultimately the samples will be acquired in polar coordinates and will need to be resampled onto a Cartesian grid of pixels for display.

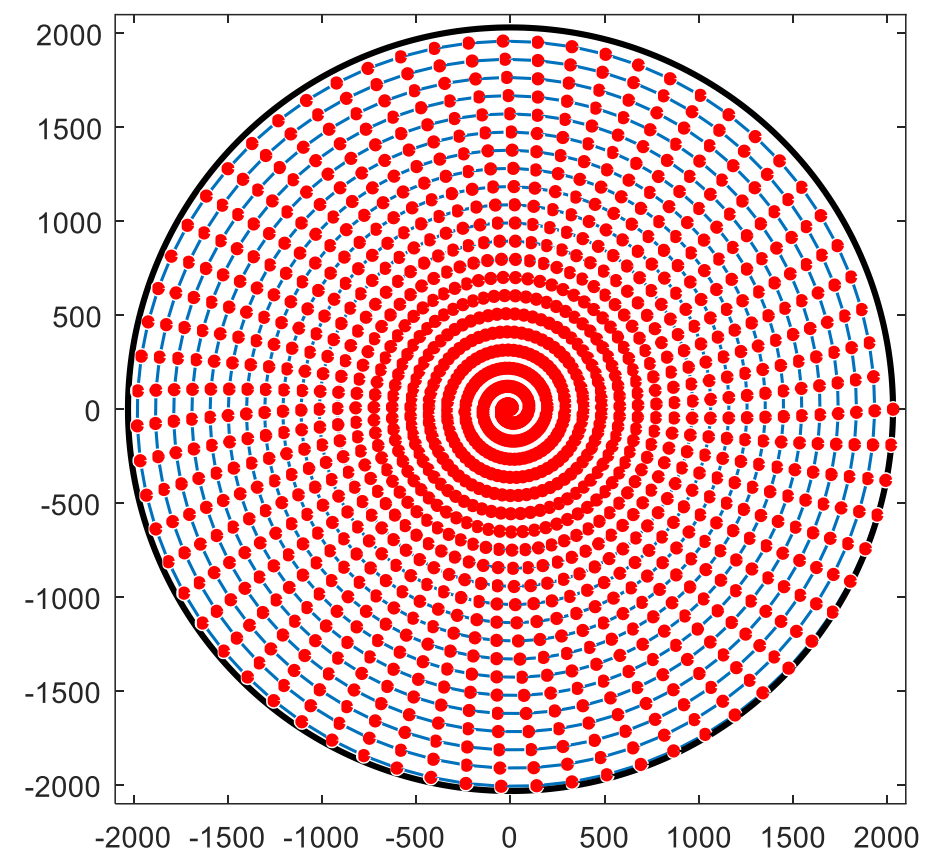

Figure 7: Sample illumination spiral scan with 21 branches. Red circles represent acquisition sample points with 67 samples per branch. The radius of the red circles is equal to the airy disk radius of 19.05 microns. The outer black circle represents the maximum image size of radius $2.032 \mathrm{~mm}$ at a working distant of $3.8 \mathrm{~mm}$. Axes have units of microns.

\section{CONCLUDING REMARKS}

The optical designs presented here may have significant utility for future tubal endoscopy applications such as imaging inside of the fallopian tube, bile ducts and cardiovascular system. Experimental verification remains necessary to validate some of the theoretical work. The properties of SM fibers designed for OCT when used out of specification for visible wavelengths needs evaluation. Fusion spliced no core and GRIN fiber has been successfully used for OCT focusing optics in the past ${ }^{8-10}$, but the utility for increasing SF divergence must be validated. GRIN fibers are sometimes used for mode scrambling of multimode fibers so the use of a GRIN element for divergence increase will alter the illumination quality of the visible beams in the OCT SM fiber. Most importantly, fabrication, coating and testing the DRCP needs completion

The DRCP is modeled using acrylic for fabrications using diamond turning in order to allow different annular surface profiles. The physical limits of the diamond turning tool will determine the maximum possible slope and depth of the concave surface of the DRCP. A larger version of the DRCP should be designed and fabricated for benchtop proof-ofconcept testing. The design offers a promising approach to using SF technology for side-viewing OCT. One limitation of the current design is the limited FFOV of the forward channel due to the spatial separation of the beam paths.

An alternate approach may be spectral separation of the near IR OCT beam from the forward-viewing visible channel. The first reflective surface could use a dichroic coating to reflect the OCT beam and transmit the navigational beam. This would potentially allow for the navigational channel to use the full spatial extent of the optical element increasing 
the FFOV. The approach may also allow for an overall size reduction necessary for endoscopes with outer diameter of approximately $1 \mathrm{~mm}$.

The DRCP complicates the light collection geometry and further design work is necessary to determine the best collection solution. Placing the collection fibers behind the cover plate may allow back reflections from the proximal face of the cover plate to enter the collection fibers. Additionally, the annular mirrored surface of the cover plate will partially obscure the collection cone of each fiber. An alternative is to place the ring of collection fibers outside the DRCP with the proximal faces of the DRCP and collection fibers aligned at the proximal end of the endoscope tip. This approach solves the aforementioned issues for the forward viewing channel, but requires the OCT beam to pass through the ring of collection fibers. The collection fiber materials could be chosen to be IR transmissive, but the ring of fibers will still induce aberrations and back reflections into the OCT path. The diameter of the endoscope will be increased leading to a reduced working distance of the side-viewing OCT channel. It may be possible to use a custom capillary tube with a hollow core as an annular waveguide ${ }^{11}$. A short length of rigid capillary tube could be placed around the proximal end of the rigid tip including the DRCP. The OCT beam would still need to pass through the waveguide, but the optical power would be rotationally symmetric and could be compensated for in the design of the DRCP. A ring of collection fibers could be placed at the proximal end of the waveguide in order to transmit light through the flexible region of the endoscope back to the proximal systems. As with the ring of collection fibers outside the DRCP the approach may increase the diameter of the endoscope, but still warrants further consideration if placing the collection fibers behind the DRCP proves to be an issue.

After initial proof of concept testing the design can be modified in terms of size and optical characteristics for a specific application. This could include the addition of illumination wavelengths for color or multispectral fluorescence imaging.

\section{REFERENCES}

[1] Lee, C. M., Engelbrecht, C. J., Soper, T. D., Helmchen, F.., Seibel, E. J., "Scanning fiber endoscopy with highly flexible, 1-mm catheterscopes for wide-field, full-color imaging," J. Biophotonics 3(5-6), 385-407 (2010).

[2] Huo, L., Xi, J., Wu, Y.., Li, X., "Forward-viewing resonant fiber-optic scanning endoscope of appropriate scanning speed for 3D OCT imaging," Opt. Express 18(14), 14375-14384 (2010).

[3] Park, H.-C., Seo, Y.-H.., Jeong, K.-H., "Lissajous fiber scanning for forward viewing optical endomicroscopy using asymmetric stiffness modulation," Opt. Express 22(5), 5818-5825 (2014).

[4] Park, H.-C., Song, C., Kang, M., Jeong, Y.., Jeong, K.-H., "Forward imaging OCT endoscopic catheter based on MEMS lens scanning," Opt. Lett. 37(13), 2673-2675 (2012).

[5] Marcuse, D., "Loss analysis of single-mode fiber splices," Bell Syst. Tech. J. 56(5), 703-718 (1977).

[6] Parulekar, S. G., "Ultrasound evaluation of common bile duct size," Radiology 133(3 Pt 1), 703-707 (1979).

[7] "Optimization Online - Solving the problem of packing equal and unequal circles in a circular container.", <http://www.optimization-online.org/DB_HTML/2008/06/1999.html> (5 January 2017 ).

[8] Keenan, M., Tate, T. H., Kieu, K., Black, J. F., Utzinger, U.., Barton, J. K., "Design and characterization of a combined OCT and wide field imaging falloposcope for ovarian cancer detection,” Biomed. Opt. Express 8(1), 124-136 (2017).

[9] Lorenser, D., Yang, X., Kirk, R. W., Quirk, B. C., McLaughlin, R. A.., Sampson, D. D., "Ultrathin side-viewing needle probe for optical coherence tomography," Opt. Lett. 36(19), 3894-3896 (2011).

[10] McLaughlin, R. A., Lorenser, D.., Sampson, D. D., "Needle Probes in Optical Coherence Tomography," Handbook of Coherent-Domain Optical Methods: Biomedical Diagnostics, Environmental Monitoring, and Materials Science, V. V. Tuchin, Ed., Springer New York, New York, NY, 1065-1102 (2013).

[11] Stasio, N., Shibukawa, A., Papadopoulos, I. N., Farahi, S., Simandoux, O., Huignard, J.-P., Bossy, E., Moser, C.., Psaltis, D., "Towards new applications using capillary waveguides," Biomed. Opt. Express 6(12), 46194631 (2015). 